\title{
ARTICLES
}

\section{Differential Effects of Phenylalanine on Rac1, Cdc42, and RhoA Expression and Activity in Cultured Cortical Neurons}

\author{
YONGJUN ZHANG, HUIWEN ZHANG, XIAOBING YUAN, AND XUEFAN GU

\begin{abstract}
XinHua Hospital [Y.Z., H.Z., X.G.], Shanghai Institute for Pediatric Research, Shanghai Jiaotong University School of Medicine, Shanghai, 200092, China; Institute of Neuroscience [X.Y.], Chinese Academy of Sciences, Shanghai, 200031, China
\end{abstract}

\begin{abstract}
Phenylketonuria (PKU) is characterized by a high concentration of phenylalanine, which can lead to mental retardation. One of the characteristic pathologic changes in untreated phenylketonuria patients is a reduction in the number of axons, dendrites, and synapses in the brain. This is thought to be due to the toxic effects of phenylalanine and/or its metabolites, however, the underlying mechanism remains unclear. In this study, we observed that phenylalanine reduced the number of dendrites and dendritic spines in cultured neurons. We further demonstrated that phenylalanine down-regulated Rac1, Cdc42, and RhoA mRNA and protein expression. Pull-down assays indicated that phenylalanine caused a decrease in Rac1/Cdc42 activity but increased RhoA activity. Expression of a dominant negative RhoA or treatment with a Rho-associated kinase specific inhibitor, Y-27632, partly inhibited the phenylalanine-induced decrease in dendrite numbers. In conclusion, we have demonstrated that phenylalanine affects the expression and activity of Rac1, Cdc42, and RhoA. Furthermore, RhoA signaling is involved in the inhibitory effect of phenylalanine on dendritic branching. These results may provide an important insight into the molecular mechanism underlying phenylalanine-induced abnormalities of dendrites, specifically in phenylketonuria neuronal injury. (Pediatr Res 62: 8-13, 2007)
\end{abstract}

$\mathrm{P}$ henylketonuria $(\mathrm{PKU})$ is one of the most frequent inborn disorders of amino acid metabolism. It is caused by a deficiency in phenylalanine hydroxylase $(\mathrm{PAH})$, resulting in an accumulation of its upstream metabolite phenylalanine, mainly in brain tissue and the cerebrospinal fluid of PKU patients. Phenylalanine interferes with brain development and results in severe MR, microcephaly, epilepsy, and behavioral problems. These symptoms appear to be the direct consequence of neuronal cell loss, white matter abnormalities, dendritic simplification, and synaptic density reduction (1-5). Previous studies demonstrated that phenylalanine reduced $\mathrm{Na}+, \mathrm{K}+$-ATPase and creatine kinase activity in rat brain (6-8). Moreover, some neurotransmitters such as serotonin and dopamine were deficient in PKU animal models (9). Glushakov et al. (10) found that a high

Received October 6, 2006; accepted February 24, 2007

Correspondence: Xuefan Gu, Ph.D., XinHua Hospital, Shanghai Institute for Pediatric Research, Department of Endocrinology and Genetic Metabolism, Kongjiang Road 1665\#, Shanghai 200092, China; e-mail: guxuefan@online.sh.cn

This work was supported by grant no. 30340082 to X.F.G and in part by grant no. 30670797 to Y.J.Z from the National Natural Science Foundation of China. concentration of phenylalanine attenuated NMDA-activated currents in cultured hippocampal neurons. In vivo ${ }^{31} \mathrm{P}-\mathrm{MRS}$ demonstrated abnormalities of cerebral energy metabolism in PKU, indicating a link between phenylalanine neurotoxicity and imbalances of cerebral energy metabolism (11). Lately, Horster et al. (5) revealed that phenylalanine reduced synaptic density and simultaneously inhibited pyruvate kinase in mixed cortical cultures. Our laboratory reported that phenylalanine and its metabolites caused neuronal cell apoptosis in vitro $(12,13)$. Although these observations demonstrate that phenylalanine is probably the main causative agent of brain injury in PKU, the pathophysiological mechanisms of its actions in brain injury are multiple and not fully understood.

As dendrites are the site of most synaptic contacts, dendritic development determines the number and pattern of synapses received by each neuron. Consequently, the defects in dendritic growth are profound, often accompanied by severe neurodevelopment disorders (14,15). Early experiments demonstrated that Down's, Ret, fraX, and fetal alcohol syndrome were associated with a reduction in dendritic spines and dendritic branches. Similar abnormalities were found in PKU, suggesting that MR was in part due to an aberrant development of connectivity $(16,17)$. Thus, the proper growth and arborization of dendrites are crucial for normal nervous system function. Although these observations indicate that synaptic remodeling and dendritic plasticity are important for cognitive functions, the underlying mechanisms of abnormal dendritic development are still unclear. It is likely that the molecules involved would have the ability to influence the actin cytoskeleton, because dendritic growth and remodeling involve major cytoskeletal reorganization. Members of the Rho family of small GTPases, Rac1, Cdc42, and RhoA, are centrally involved in cytoskeletal reorganization and known to regulate neuronal morphogenesis, including migration, polarity, axon growth and guidance, dendritic elaboration and plasticity, and synapse formation $(18,19)$. Mutations in Rholinked genes, such as OPHN1, PAK3, and ARHGEF6, have been reported to result in neuronal connectivity deficiencies that give rise to MR (17). Thus, it is likely that Rho GTPases

Abbreviations: MR, mental retardation; PKU, phenylketonuria; ROCK, Rho-associated kinase 
are involved in MR and phenylalanine probably has effects on Rho GTPases. We hypothesized that phenylalanine might affect Rac1, Cdc42, and RhoA expression and activity, and thereby disturb dendritic development.

\section{METHODS}

Neuronal culture. The use and care of animals followed the guideline of the Shanghai Institutes for Biologic Sciences Animal Research Advisory Committee. SD rats were deeply anaesthetized by injection of sodium pentobarbital (100 mg / kg body weight). Cortical neurons were prepared from 14-d-old rat embryos by methods described previously (13) with some modifications. Briefly, culture medium consisted of Neurobasal (Invitrogen, Carlsbad, CA.), 2\% B27 (Invitrogen), and 0.5-mM glutamine (Invitrogen). Cortical neurons were plated on Poly-D-lysine coated dishes or coverslips. For some experiments, $0.9 \mathrm{mM}$ phenylalanine (Sigma Chemical Co., St. Louis, MO) or $10 \mu \mathrm{M}$ Y-27632 (Calbiochem, San Diego, CA) was added into the medium if necessary.

Immunocytochemistry. Cultured cortical neurons were fixed with $4 \%$ paraformaldehyde in PBS for 20 min at $4{ }^{\circ} \mathrm{C}$, and then washed with PBS three times for 10 min duration per wash. After permeabilization with $0.1 \%$ triton X-100 in PBS for 10 min, nonspecific binding was blocked with $10 \%$ sheep serum in PBS for $1 \mathrm{~h}$ at $37^{\circ} \mathrm{C}$ followed by primary antibody incubation for $12 \mathrm{~h}$ at $4^{\circ} \mathrm{C}$. Neurons were rinsed three times (10 min each) in PBS and incubated for $1 \mathrm{~h}$ at room temperature with secondary antibody. PBS washing was applied three times before visualization under a fluorescent microscope.

Assay for dendritic growth. Neurons were fixed with $4 \%$ paraformaldehyde, probed with primary antibodies rabbit anti-MAP-2 polyclonal antibody (Chemicon International, Temecula, CA) and mouse anti-Tau-1 MAb (Chemicon International), and double-immunostained with secondary antibodies goat anti-mouse IgG $(\mathrm{H}+\mathrm{L})(488 \mathrm{~nm}$, green fluorescence, Jackson ImmunoResearch, West Grove, PA) and goat anti-rabbit IgG $(\mathrm{H}+\mathrm{L})(546 \mathrm{~nm}$, red fluorescence, Jackson ImmunoResearch, USA). Images were acquired with a Nikon fluorescence microscopy $(40 \times)$. Primary dendrites were considered when processes extended directly from the cell body, and dendritic branches were considered when processes branched off primary dendrites. The number of primary dendrites and dendritic branches were counted with Neurolucida 6.0 Soft.

Spine density analysis. Spine density was assessed in fixed neurons immunostained with anti-MAP-2. Confocal images were obtained using a Zeiss confocal microscope (100×, NA 1.30, $546 \mathrm{~nm}$ laser, LSM 510). Optical serial sections of $0.5 \mu \mathrm{m}$ were taken through the cells and reconstructed to yield a complete "three-dimensional" image of individual cells in focus. Spines were defined as being 1-5 $\mu \mathrm{m}$ in length. Spine density [number of spines per unit length $(10 \mu \mathrm{m})$ ] was calculated with Neurolucida 6.0 Soft.

RNA extraction. Total RNA was isolated from cortical neurons using an RNA extraction reagent, TRIzol (Invitrogen, Carlsbad, CA), according to the manufacturer's instruction.

Real-time PCR. Real-time PCR was performed with the LightCycler thermal cycling system (Roche Diagnostics, Indianapolis, IN) using the RNA Master SYBR Green I kit (Roche Diagnostics, Mannhein, Germany) as described by the manufacturer. PCR amplification was initiated with an incubation at $95^{\circ} \mathrm{C}$ for $30 \mathrm{~s}$, followed by 35 cycles of $95^{\circ} \mathrm{C}$ for $10 \mathrm{~s}, 62^{\circ} \mathrm{C}$ ( $\beta$-actin), $64^{\circ} \mathrm{C}(\mathrm{Rac} 1), 65^{\circ} \mathrm{C}(\mathrm{Cdc} 42), 66^{\circ} \mathrm{C}(\mathrm{RhoA})$ for $5 \mathrm{~s}$, and $72^{\circ} \mathrm{C}$ for $10 \mathrm{~s}$. Rac1 primer sequences were $5^{\prime}$-caa tgc gtt ccc tgg aga gta ca-3' (sense) and $5^{\prime}$ - acg tct gtt tgc ggg tag gag ag $-3^{\prime}$ (antisense). Cdc 42 primer sequences were $5^{\prime}$ - taa ctc acc act gtc caa aga ctc c- $3^{\prime}$ (sense) and $5^{\prime}$-cct cat caa aca cat tct tca gac c- $3^{\prime}$ (antisense). RhoA primer sequences were $5^{\prime}$ - cca aat gtg ccc atc atc cta gtt g- $3^{\prime}$ (sense) and $5^{\prime}$ - tcc gtc ttt ggt ctt tgc tga aca c- $3^{\prime}$ (antisense). The primers for $\beta$-actin were $5^{\prime}$-aga cct cta tgc caa cac agt gct g- $3^{\prime}$ (sense) and $5^{\prime}$-tca tcg tac tcc tgc ttg ctg a $-3^{\prime}$ (antisense). Primers were synthesized by Shanghai Sangon Co, Ltd. The Second Derivate Maximum Method was performed for CP (crossing point) determination, using LightCycler Software 3.5 (Roche Molecular Biochemicals, Indianapolis, IN). The specificity of the amplification reactions was confirmed by analyzing their corresponding melting curves. Real-time PCR amplification efficiencies $(E)$ were calculated from the slopes given in LightCycler software 3.5. The relative expression ratio of a target gene was calculated based on $E$ and the CT (cycle threshold) deviation of the sample versus control, and expressed in comparison to a reference gene (20).

Western blotting. Cells were lysed in lysis buffer $(0.1 \%$ SDS, $1 \%$ NP-40, $50 \mathrm{mM}$ HEPES, $\mathrm{pH}$ 7.4, $2 \mathrm{mM}$ EDTA, $100 \mathrm{mM} \mathrm{NaCl}, 5 \mathrm{mM}$ sodium orthovanadate, $40 \mu \mathrm{M}$ p-nitrophenyl phosphate, and $1 \%$ protease inhibitor mixture set I; Calbiochem, San Diego, CA). The lysates were centrifuged at $12,000 \mathrm{rpm}$ for $25 \mathrm{~min}$. The supernatant was collected and denatured. Samples for electrophoresis were solubilized with the loading buffer $[2.5 \mathrm{mM}$ Tris $(\mathrm{pH}$
6.8), $25 \%$ glycerol, $2 \%$ SDS, $5 \%$ 2-mercaptoethanol, $0.05 \%$ bromophenol blue], heated to $100^{\circ} \mathrm{C}$ for $5 \mathrm{~min}$. Equalized amounts of proteins were subjected to $15 \%$ SDS-PAGE gel and blotted onto a polyvinylidene diflouride membrane. The blot was blocked for $4-5 \mathrm{~h}$ at room temperature in $0.2 \%$ block-1 (Applied Biosystems, Foster City, CA), followed by incubation overnight at $4^{\circ} \mathrm{C}$ with primary antibodies $(1: 1000$, mouse MAb against Rac1 and Cdc42 from Upstate Group, Waltham, MA; mouse MAb against RhoA from Santa Cruz Biotechnology, Santa Cruz, CA). Membranes were rinsed and incubated for $1 \mathrm{~h}$ with peroxidase-conjugated goat anti-mouse $\operatorname{IgG}$ (1:10,000; Bio-Rad, Hercules, CA). Chemiluminescent detection was performed with the ECL kit (Pierce, Rockford, IL). Actin (Mouse MAb, Chemicon International) was used as loading control.

Assay for GTPase activity. We measured GTPase activity by methods as described previously (21). Briefly, lysates of cortical neurons were preincubated with glutathione-Sepharose $4 \mathrm{~B}$ beads (Amersham Biosciences, Little Chalfont, Buckinghamshire, UK) at $4^{\circ} \mathrm{C}$ for $1 \mathrm{~h}$ with constant rocking and then centrifuged to remove the nonspecific binding. The supernatants were incubated with the bacterially produced glutathione $S$-transferase (GST)-fused Rac/Cdc42-binding domain of Pak (GST-PBD; $5 \mu \mathrm{g}$ ) or the Rho-binding domain of Rhotekin (GST-RBD; $20 \mu \mathrm{g}$ ) bound to glutathione-coupled Sepharose beads at $4^{\circ} \mathrm{C}$ for $1 \mathrm{~h}$ with constant rocking. The beads and proteins bound to the fusion protein were washed five times with lysis buffer at $4^{\circ} \mathrm{C}$, eluted in SDS sample buffer, and analyzed for bound Rac1, Cdc42, and RhoA by Western blotting using anti-Rac1, anti-Cdc42, and anti-RhoA antibodies. The quantities of Rac1, Cdc42, and RhoA activation were measured by densitometric analysis of the blots. Protein samples of 1/10th of total lysates were shown to Rac1, Cdc42, and RhoA expression by Western blotting.

Plasmid transfection dominant-negative (DN) RhoA (N19 RhoA) was described previously (21). Neuron transfection was carried out immediately after dissociation using the Amaxa Nucleofector device and kit (Amaxa Biosystems, Cologne, Germany). Briefly, dissociated cells were resuspended in transfection medium, mixed with plasmids, and electroporated using the fixed program (O-03) to yield minimal toxicity and maximal transfection efficiency. Cells were then quickly centrifuged, resuspended, and plated.

Statistical analysis. All the data in this study are expressed as mean \pm SD from at least three independent experiments. Statistical analysis was performed using one-way ANOVA or by independent-samples $t$ test. For statistical evaluations of the determined CT variations and calculated relative expression variations, data were analyzed for significant differences using relative expression software tool (REST), provided by MW Pfaffi; (20). A value of $p<0.05$ was considered statistically significant.

\section{RESULTS}

Phenylalanine treatment decreased dendrites and dendritic spines. Using morphologic analysis, we sought to determine whether phenylalanine affected dendritic growth. 0.9 $\mathrm{mM}$ phenylalanine, which was determined by measuring the concentration of phenylalanine in the cerebrospinal fluid of a PKU patient, was added to the culture medium of neurons at $3 \mathrm{~d}$ in vitro (DIV 3). Two or four days later (DIV 5, DIV 7), neurons were fixed and double-immunostained with antiMAP-2 (red) and anti-Tau-1 (green) so that the MAP-2positive but Tau-1 negative dendrite was clearly discernible. We found that phenylalanine treatment decreased the number of dendrites at DIV 5 (Fig. 1A; control, $5.011 \pm 1.772$ for primary dendrites, $1.973 \pm 1.562$ for dendrite branches; Phe, $4.199 \pm 1.671$ for primary dendrites, $1.199 \pm 1.091$ for dendrite branches). Similar results were also observed at DIV 7 (Fig. 1A; control, $5.383 \pm 2.286$ for primary dendrites, $3.539 \pm 2.419$ for dendritic branches; Phe, $4.637 \pm 1.487$ for primary dendrites, $2.615 \pm 1.914$ for dendritic branches). We next determined whether phenylalanine had an effect on spine formation. Treatment with phenylalanine for $14 \mathrm{~d}$ (DIV 17) significantly decreased the density of dendritic spines (Fig. 1B; control, $4.931 \pm 1.331$; Phe, $3.642 \pm 0.788$ ). 

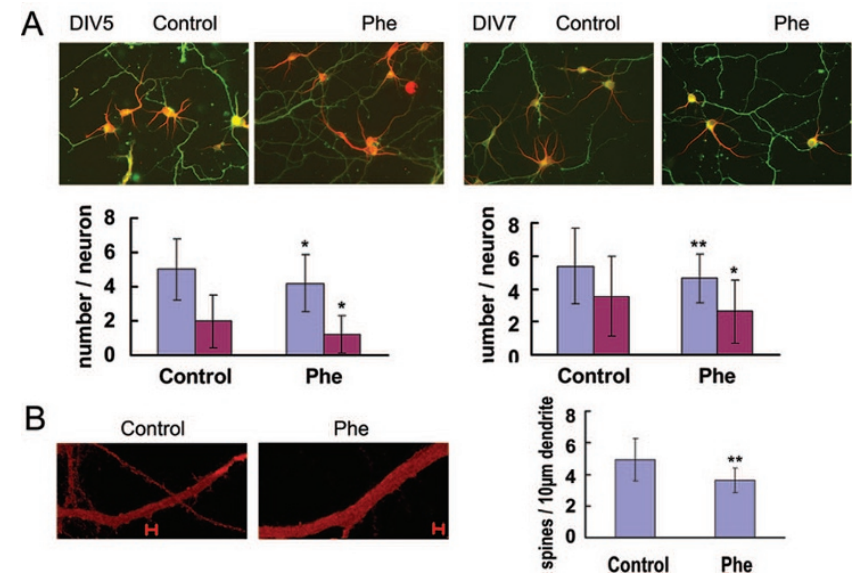

Figure 1. Reduced dendrites and dendritic spines by phenylalanine (phe) in cortical neurons. (A) Cultured neurons at $3 \mathrm{~d}$ in vitro (DIV 3) were treated with $0.9 \mathrm{mM}$ phenylalanine. Two or four days later (DIV 5, DIV 7), neurons were fixed and double-immunostained. Primary dendrites (blue columns); dendrite branches (red columns); $n=130-180$ each; * $p<0.01$, ** $p<0.05$. (B) Neurons were cultivated up to DIV 3, and then treated with $0.9 \mathrm{mM}$ phenylalanine. Fourteen later (DIV 17), cultured neurons were fixed and immunostained. $n=40-50$ each; $* * p<0.05$. Scale bars: $10 \mu \mathrm{m}$.

\section{A}

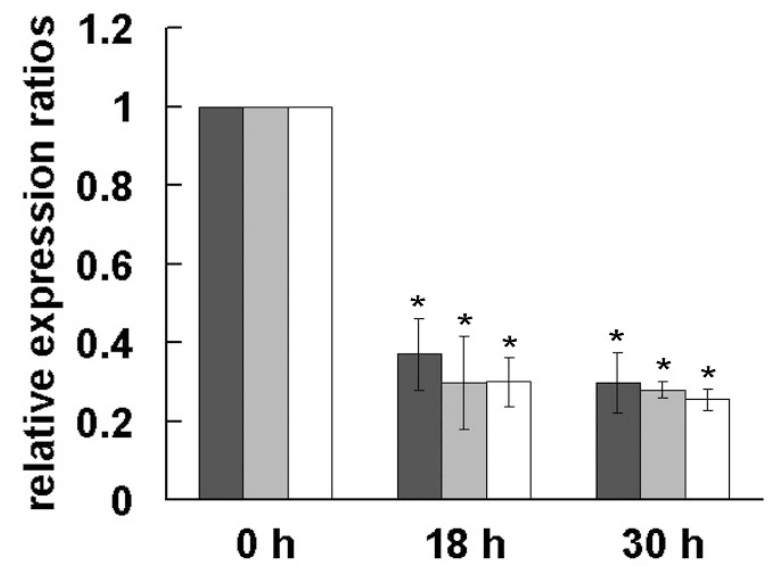

B
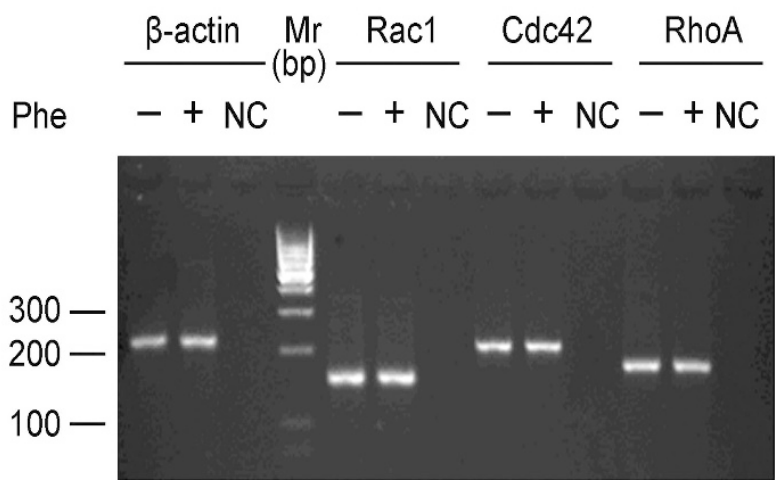

Figure 2. Down-regulation of Rac1, Cdc42, and RhoA mRNAs expression by phenylalanine $(P h e)$. (A) Neurons were treated with $0.9 \mathrm{mM}$ phenylalanine for $18 \mathrm{~h}$ and $30 \mathrm{~h}$. mRNA was isolated and used as a template for a real-time PCR assay to measure the relative levels of Rac1, Cdc42, and RhoA. Results are expressed as \% mRNA relative to the control. $(B)$ Analysis of amplified real-time PCR products by $2 \%$ agarose gel electrophoresis. The results showed that the lengths of $\beta$-actin and $\mathrm{Cdc} 42$ were between $200 \mathrm{bp}$ and 300 bp, whereas the lengths of Rac1 and RhoA were between 100 bp and 200 bp. They were consistent with the length of designed fragments. $N C$, negative control; Rac1 (black bars); Cdc42 (shaded bars); RhoA (white bars). $n=3-4$ each; *p $<0.01$
Rac1, Cdc42, and Rhoa mRNA and protein expression were down-regulated by phenylalanine. Because Rac1, Cdc42, and RhoA have been shown to regulate dendritic growth (19), we investigated whether phenylalanine affected their expression. Real-time PCR was used to study the levels of Rac1, Cdc42, and RhoA mRNA in cultured neurons. After $3 \mathrm{~d}$ culture, the cells were treated with $0.9 \mathrm{mM}$ phenylalanine for an additional $6 \mathrm{~h}, 18 \mathrm{~h}$, and $30 \mathrm{~h}$. As shown in Figure 2A, phenylalanine treatment significantly down-regulated expression of Rac1 mRNA, which was $37.0 \pm 9.1 \%$ and $29.9 \pm$ $7.6 \%$ of control after $18 \mathrm{~h}$ and $30 \mathrm{~h}$, respectively. Similarly, treatment with phenylalanine reduced Cdc 42 mRNA levels to $29.8 \pm 11.9 \%$ and $27.9 \pm 2.0 \%$ of control and RhoA mRNA levels to $30 \pm 6.4 \%$ and $25.7 \pm 2.8 \%$ of control, respectively. These data suggest that down-regulation of Rac1, Cdc42, and RhoA expression by phenylalanine is probably mediated by inhibition of gene transcription. We further analyzed Rac1, $\mathrm{Cdc} 42$, and RhoA expression at the protein level. After phenylalanine treatment, Western blot analysis showed Rac1, Cdc42, and RhoA expression were time-dependently downregulated, results consistent with the down-regulation of their respective genes (Fig. 3, $A-C$ ).

Rac1, Cdc42, and RhoA activities were affected by phenylalanine. To investigate whether phenylalanine had an effect on Rac1, Cdc42, and RhoA activities, their activities were assessed using GST pull-down assays. Our results showed that treatment with phenylalanine down-regulated Rac1 and Cdc42 activities (Fig. 3, $A$ and $B$ ). In contrast to the down-regulation of RhoA expression, RhoA was activated after phenylalanine treatment (Fig. 3C). Moreover, these changes were observed after only $6 \mathrm{~h}$ of phenylalanine exposure.

Reduced dendrite in response to phenylalanine was mediated by RhoA signaling. Next, we investigated whether the Rho GTPases were involved in phenylalanine-induced inhibition of dendritic branching. We first examined the effect of expressing a dominant negative form of RhoA. Three days after transfection, cells were treated with phenylalanine and dendrite numbers were recorded $2 \mathrm{~d}$ later (DIV 5). Expression of DN-RhoA increased the number of dendrites compared with control vector. Specifically, there was no significant difference between DN-RhoA group and DN-RhoA/Phe group. This indicated that expression of DN-RhoA partially inhibited the phenylalanine-induced decrease in dendrites (Fig. 4A; GFP, $3.967 \pm 1.438$ for primary dendrites, $2.05 \pm$ 1.534 for dendrite branches; GFP/Phe, $3.133 \pm 1.157$ for primary dendrites, $1.4 \pm 1.355$ for dendrite branches; DN RhoA, $4.889 \pm 1.764$ for primary dendrites, $3.556 \pm 1.944$ for dendrite branches; DN RhoA/Phe $4.8 \pm 1.398$ for primary dendrites, $2.8 \pm 1.619$ for dendrite branches). In our study, with the electro-transfection method we were not able to generate expression of a constitutively active Rac1 and Cdc42 in immature neurons, most likely because active forms of these proteins are toxic.

RhoA initiates cellular processes by acting on ROCK, a direct downstream effector (22). ROCK has been shown to be particularly important in neuronal morphogenesis. This was demonstrated in several studies that had shown that RhoAmediated neurite retraction could be prevented by inhibition of 
A
Time (hr)

B

Time (hr) $\quad \begin{array}{llll}0 & 6 & 18 & 30\end{array}$
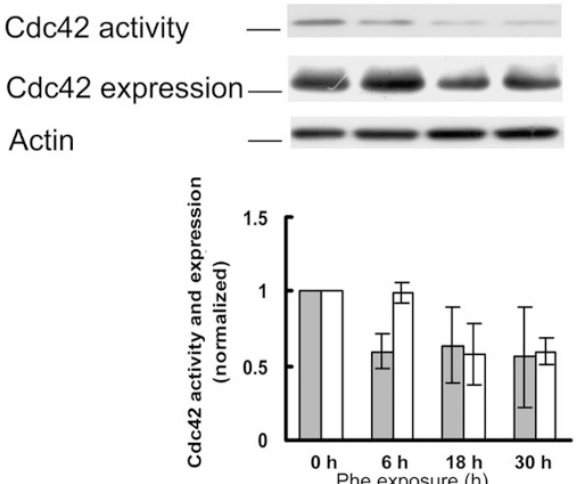

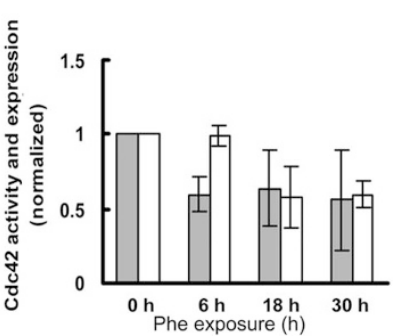

C

$$
\text { Time (hr) } \quad 0 \quad 6 \quad 18 \quad 30
$$
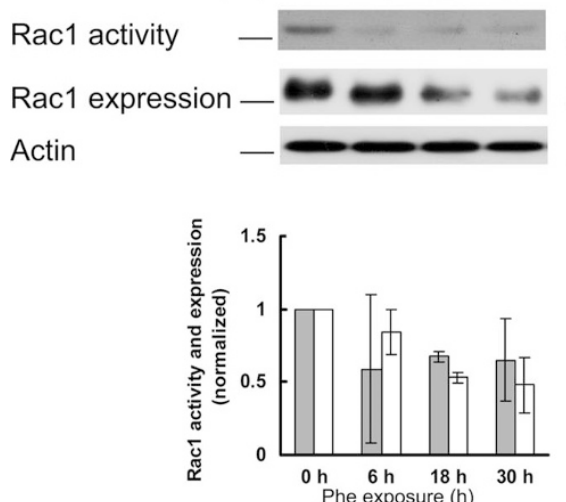

Figure 3. Effects of phenylalanine (Phe) on the Rac1, Cdc42 and RhoA protein expression and activity. Neurons were treated with $0.9 \mathrm{mM}$ phenylalanine for the indicated times. The cell lysates were incubated with GST-PBD and GST-RBD, and the amounts of active GTPases were determined by Western blotting. The protein expressions of Rac1, Cdc42, and RhoA in cell lysates were also shown. (A) Down-regulation of Rac1 protein expression and activity by phenylalanine. $(B)$ Down-regulation of $\mathrm{Cdc} 42$ protein expression and activity by phenylalanine. $(C)$ Phenylalanine down-regulated RhoA protein expression but increased its activity. Rac1, Cdc42, and RhoA activity are normalized to their expression levels, and their expressions are normalized to loading control in the same sample. Values of Rac1, Cdc42, and RhoA activity and protein expression are expressed as relative to or -fold increase over the value of the control. Rac1, Cdc42, and RhoA activity (shaded bars); Rac1, Cdc42, and RhoA expression (white bars). $n=4-5$ each.

ROCK activity $(23,24)$. To further analyze that RhoA signaling is involved in phenylalanine-induced dendritic simplification, we used Y-27632, a ROCK-specific inhibitor, to determine whether the phenylalanine-induced decrease in dendritic numbers can be reversed when ROCK activity is blocked. Three-day-old cultured neurons were pretreated with Y-27632 $(10 \mu \mathrm{M})$ for $2 \mathrm{~h}$ and then treated with phenylalanine for $2 \mathrm{~d}$ (DIV 5). Phenylalanine decreased the number of dendrites, which was restored by preincubation with Y-27632 (Fig. 4B; control, $4.786 \pm 1.623$ for primary dendrites, $2.054 \pm 1.725$ for dendrite branches; Phe, $3.855 \pm 1.436$ for primary dendrites, $1.244 \pm 1.103$ for dendrite branches; Y-27632, $4.987 \pm$ 1.728 for primary dendrites, $2.032 \pm 1.331$ for dendrite branches; Y-27632/Phe $5.095 \pm 1.606$ for primary dendrites, $1.869 \pm$ 1.187 for dendrite branches). These data indicate that RhoA signaling may be partially responsible for the inhibitory effect of phenylalanine on dendritic branching.

\section{DISCUSSION}

Abnormalities in dendritic morphology have been described in PKU brains. Robain et al. (25) reported a reduction in the number of dendritic branches in cerebellar Purkinje cells in the PKU rat. Cordero et al. (26) found similar changes in the basal dendritic trees of large pyramidal neurons. Lacey (27) found spars and malformed dendritic spines on layer 5 pyramidal cells. Williams et al. (28) described a decrease in dendritic spins on cortical pyramidal cell in PKU patients. Decreased synaptogenesis in the cerebral cortex of the PKU rat has also been shown (29). An autopsy study of an untreated patient reported neuronal loss, reduced neuronal size, and decreased dendritic processes of Purkinje cells (30). Recent evidence demonstrated a decreased synaptic density in phenylalanine-treated cortical cultures, with no changes in dendritic morphology (5). In our neuronal cultures, we observed that phenylalanine induced a decrease in the number of dendrites and dendritic spines, suggesting a decrease in axo- dendritic synaptic contacts. However, some reports have been inconsistent with these observations. For example, there have been reports of an increase in the density of dendritic spines in hippocampal neurons of rat made hyperphenylalaninemic (31). These differences may be due to different quantitative methods, or the use of neuron at different developmental stages. It was concluded that phenylalanine interfered with dendritic and synaptic development.

As it is clear that Rho GTPases have important effects on the actin cytoskeleton, their involvement in nervous system development has been previously characterized $(18,19)$. It is possible that the alteration of these enzymes may impair neuronal development in PKU, thereby contributing to the abnormalities in axons, dendrites, and synapses. At present, our knowledge of the role of Rho GTPases in the development, plasticity, and function of the nervous system is fragmented and sometimes contradictory. Studies examining expression of different members of the Rho GTPase family in cultured cortical neurons demonstrated that Rho GTPases were likely to function in the cortex throughout development and were also present during periods of axonal and dendritic growth. Rac1 expression was up-regulated during the early stage of neurite outgrowth, whereas RhoA expression was up-regulated during later stages, possibly during synaptogenesis. Furthermore, the number of dendrites depended on Rac1, Cdc42, and RhoA expression (19,32,33). Threadgill and Ghosh (32) found that expression of dominant negative mutants of Rac and Cdc42 in cultured cortical neurons markedly reduced the number of primary dendrites. Cdc42 mutant neurons also show a reduction in dendritic spine density and defect in dendrite caliber (34). Rac1 seemed to play a preferential role in regulating spine morphogenesis. Expression of activated Rac1 in mouse Purkinje cells resulted in drastic changes in dendritic spine morphology, whereas RhoA was implicated in limiting the growth of dendritic branches $(24,35)$. However, some reports showed axonal development 


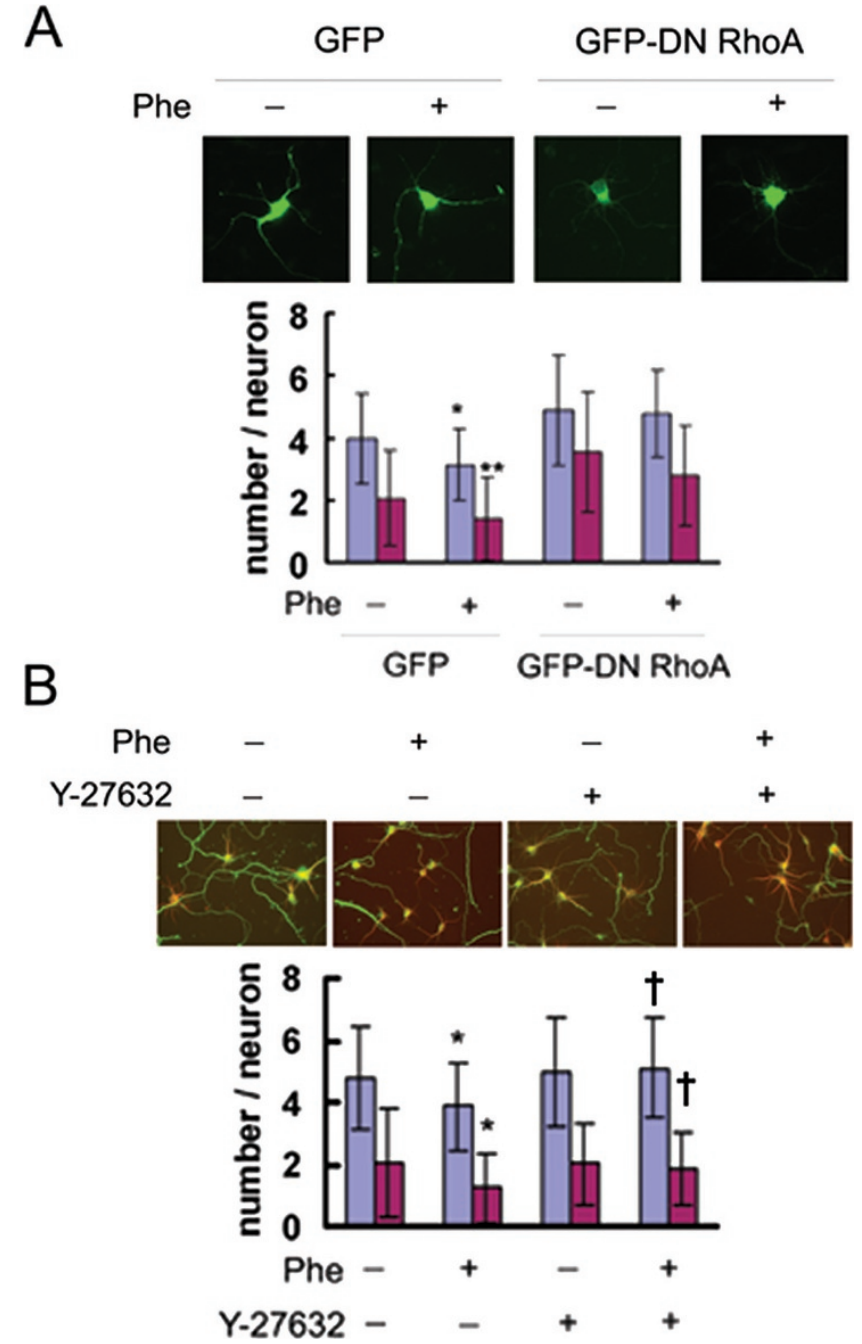

Figure 4. Inhibition of RhoA signaling attenuated phenylalanine (Phe)induced decrease in dendritic branching. (A) Neurons were transfected with either GFP-vector or GFP-DN-RhoA. Three days after transfection, cells were treated with phenylalanine $(0.9 \mathrm{mM})$ for an additional $2 \mathrm{~d}$ (DIV 5). Transfected cells were identified by GFP fluorescence. Expression of DN-RhoA increased the number of dendrites, and partly inhibited phenylalanine-induced dendritic decrease. $n=30-40$ each, ${ }^{*} p<0.01,{ }^{*} p<0.05$ compared with the GFP-vector control. $(B)$ Cultured neurons at $3 \mathrm{~d}$ were pretreated with Y-27632 $(10 \mu \mathrm{M})$ for $2 \mathrm{~h}$, then phenylalanine $(0.9 \mathrm{mM})$ was added to the cultures. Two days later (DIV 5), neurons were fixed and doubleimmunostained. Phenylalanine-induced dendritic decrease is reversed by preincubation of neurons with Y-27632. $n=200-280$ each; $* p<0.01$ compared with the control group; $\dagger p<0.01$ compared with the Phe group. ANOVA followed by post hoc test; primary dendrites (blue columns); dendrite branches (red columns).

required activation of RhoA, whereas dendritic development benefited from its inactivation (36). Activation of RhoA had been shown to promote dendritic and axonal growth in rat cortical neurons cultivated in the presence of serum (32). Thus, RhoA appears to have variable effects on all types of processes depending on the developmental stage of the neurons and the culture conditions. In our study, significant down-regulation of Rac1, Cdc42, and RhoA mRNA and protein expression was observed after $18 \mathrm{~h}$ of phenylalanine exposure. A decreased Rac1/Cdc42 activity and increased RhoA activity was observed in neurons treated with phenyl- alanine for more than $6 \mathrm{~h}$. These data suggested that phenylalanine inhibited Rac1, Cdc42, and RhoA gene transcription and might directly affect GTP levels. We speculated that alterations in Rho GTPase activity might be partly caused by a down-regulation of their expressions. In addition, Rac1, Cdc42, and RhoA can influence each other's activity: Cdc42activated Rac1 could antagonize Rho activation, and, in more limited cases, Rac1 down-regulated RhoA activity $(37,38)$, and RhoA inhibited Rac1 activation through Rho-associated kinase-dependent pathway (39). Furthermore, there is evidence of crosstalk between the Cdc42 and RhoA pathways in mediating axon guidance (21). Thus, it is likely that phenylalanine-induced Rac1, Cdc42, and RhoA activity is coregulatory.

This study has demonstrated that expression of dominantnegative forms of RhoA or suppression of the RhoA downstream effector Rho kinase reverses phenylalanine-induced dendritic simplification, demonstrating that activity of the RhoA pathway may be responsible for the phenylalanineinduced changes in dendritic branching. Although phenylalanine affected the activity of Rac1/Cdc42, we could not determine whether Rac1/Cdc42 activity was important for the changes of dendrites by phenylalanine. According to previous studies $(21,24,34,35)$, it is possible that phenylalanine-induced inhibition of Rac/Cdc42 activity is involved in the development of dendritic spines or synapses and neurite growth, although no direct evidence is forthcoming.

In summary, our results revealed decreased dendrites and dendritic spines in phenylalanine-induced cortical neurons. Furthermore, we have shown that phenylalanine affected Rac1, Cdc42 and RhoA expression and activity. In addition, phenylalanine reduced dendritic branching of the cortical neurons, which was mediated by Rho signaling. However, the biochemical and cellular mechanisms underlying impaired brain development and cerebral dysfunction by phenylalanine "toxicity" are complicated, the actin skeleton and related signaling represent only a small proportion of the mechanisms that determine the development of cognitive deficits underlying PKU.

Acknowledgments. The authors thank Dr. Betty Pat for critical revision of the manuscript.

\section{REFERENCES}

1. Moller HE, Weglage J, Bick U, Wiedermann D, Feldmann R, Ullrich K 2003 Brain imaging and proton magnetic resonance spectroscopy in patients with phenylketonuria. Pediatrics 112:1580-1583

2. Huttenlocher PR 2000 The neuropathology of phenylketonuria human and animal studies. Eur J Pediatr 159:s102-s106

3. Kahler SG, Fahey MC 2003 Metabolic disorders and mental retardation. Am J Med Genet C Semin Med Genet 117:31-41

4. Bauman ML, Kemper TL 1982 Morphologic and histoanatomic observations of the brain in untreated human phenylketonuria. Acta Neuropathol (Berl) 58:55-63

5. Horster F, Schwab MA, Sauer SW, Pietz J, Hoffmann GF, Okun JG, Kolker S, Kins S 2006 Phenylalanine reduces synaptic density in mixed cortical cultures from mice. Pediatr Res 59:544-548

6. Schulpis KH, Tjamouranis J, Karikas GA, Michelakakis H, Tsakiris S 2002 In vivo effects of high phenylalanine blood levels on $\mathrm{Na}+, \mathrm{K}+$-ATPase, $\mathrm{Mg} 2+$-ATPase activities and biogenic amine concentrations in phenylketonuria. Clin Biochem 35:281-285

7. Tsakiris S 2001 Effects of L-phenylalanine on acetylcholinesterase and $\mathrm{Na}+$ $\mathrm{K}+$-ATPase activities in adult and aged rat brain. Mech Ageing Dev 122:491-501

8. Costabeber E, Kessler A, Severo Dutra-Filho C, De Souza Wyse AT, Wajner M, Wannmacher CM 2003 Hyperphenylalaninemia reduces creatine kinase activity in the cerebral cortex of rats. Int J Dev Neurosci 21:111-116 
9. Pascucci T, Ventura R, Puglisi-Allegra S, Cabib S 2002 Deficits in brain serotonin synthesis in a genetic mouse model of phenylketonuria. Neuroreport 13:2561-2564

10. Glushakov AV, Dennis DM, Morey TE, Sumners C, Cucchiara RF, Seubert CN, Martynyuk AE 2002 Specific inhibition of N-methyl-D-aspartate receptor function in rat hippocampal neurons by L-phenylalanine at concentrations observed during phenylketonuria. Mol Psychiatry 7:359-367

11. Pietz J, Rupp A, Ebinger F, Rating D, Mayatepek E, Boesch C, Kreis R 2003 Cerebral energy metabolism in phenylketonuria: findings by quantitative in vivo $31 \mathrm{P}$ MR spectroscopy. Pediatr Res 53:654-662

12. Yang XW, Gu XF, Chen RG 2000 Toxic effects of phenylacetic acid on cultured rat cortical neurons. Chinese J Neurosci 16:330-332

13. Zhang H, Gu XF 2005 A study of gene expression profiles of cultured embryonic rat neurons induced by phenylalanine. Metab Brain Dis 20:61-72

14. Hausser M, Spruston N, Stuart GJ 2000 Diversity and dynamics of dendritic signaling. Science 290:739-744

15. Matus A 2000 Actin-based plasticity in dendritic spines. Science 290:754-758

16. Kaufmann WE, Moser HW 2000 Dendritic anomalies in disorders associated with mental retardation. Cereb Cortex 10:981-991

17. Ramakers GJ 2002 Rho proteins, mental retardation and the cellular basis of cognition. Trends Neurosci 25:191-199

18. Hall A 1998 Rho GTPases and the actin cytoskeleton. Science 279:509-514

19. Luo L 2000 Rho GTPases in neuronal morphogenesis. Nat Rev Neurosci 1:173-180

20. Pfaffl MW, Horgan GW, Dempfle L 2002 Relative expression software tool (REST) for group-wise comparison and statistical analysis of relative expression results in real-time PCR. Nucleic Acids Res 30:e36

21. Yuan XB, Jin M, Xu X, Song YQ, Wu CP, Poo MM, Duan SS 2003 Signalling and crosstalk of Rho GTPases in mediating axon guidance. Nat Cell Biol 5:38-45

22. Katoh H, Aoki J, Ichikawa A, Negishi M 1998 p160 RhoA-binding kinase ROKalpha induces neurite retraction. J Biol Chem 273:2489-2492

23. Bito H, Furuyashiki T, Ishihara H, Shibasaki Y, Ohashi K, Mizuno K, Maekawa M, Ishizaki T, Narumiya S 2000 A critical role for a Rho associated kinase, p160ROCK, in determining axon outgrowth in mammalian CNS neurons. Neuron 26:431-441

24. Nakayama AY, Harms MB, Luo L 2000 Small GTPases Rac and Rho in the maintenance of dendritic spines and branches in hippocampal pyramidal neurons. J Neurosci 20:5329-5338

25. Robain O, Wen GY, Wisniewski HM, Shek JW, Loo YH 1981 Purkinje cell dendritic development in experimental phenylketonuria A quantitative analysis. Acta Neuropathol (Berl) 53:107-112
26. Cordero ME, Trejo M, Colombo M, Aranda V 1983 Histological maturation of the neocortex in phenylketonuric rats. Early Hum Dev 8:157-173

27. Lacey DJ 1986 Cortical dendritic spine loss in rat pups whose mothers were prenatally injected with phenylacetate ('maternal PKU' model). Brain Res 392:283-285

28. Williams RS, Hauser SL, Purpura DP, DeLong GR, Swisher CN 1980 Autism and mental retardation: neuropathologic studies performed in four retarded persons with autistic behavior. Arch Neurol 37:749-753

29. Loo YH, Fulton T, Miller K, Wisniewski HM 1980 Phenylacetate and brain dysfunction in experimental phenylketonuria: synaptic development. Life Sci 27:1283-1290

30. Kornguth S, Gilbert-Barness E, Langer E, Hegstrand L 1992 Golgi-Kopsch silver study of the brain of a patient with untreated phenylketonuria, seizures and cortical blindness. Am J Med Genet 44:443-448

31. Lacey DJ 1984 Hippocampal dendritic abnormalities in a rat model of Phenylketonuria. Ann Neurol 16:577-580

32. Threadgill R, Bobb K, Ghosh A 1997 Regulation of dendritic growth and remodeling by rho, rac, and cdc42. Neuron 19:625-634

33. Ramakers GJ 2000 Rho proteins and the cellular mechanisms of mental retardation. Am J Med Genet 94:367-371

34. Scott EK, Reuter JE, Luo L 2003 Small GTPase Cdc42 is required for multiple aspects of dendritic morphogenesis. J Neurosci 23:3118-3123

35. Luo L, Hensch TK, Ackerman L, Barbel S, Jan LY, Jan YN 1996 Differential effects of the Rac GTPase on Purkinje cell axons and dendritic trunks and spines. Nature 379:837-840

36. Ahnert-Hilger G, Holtje M, Grosse G, Pickert G, Mucke C, Nixdorf-Bergweiler B, Boquet P, Hofmann F, Just I 2004 Differential effects of Rho GTPases on axonal and dendritic development in hippocampal neurons. J Neurochem 90:9-18

37. Nobes CD, Hall A 1995 Rho, rac, and cdc42 GTPases regulate the assembly of multimolecular focal complexes associated with actin stress fibers, lamellipodia, and filopodia. Cell 81:53-62

38. Sander EE, Ten Klooster JP, Van Delft S, Van der Kammen RA, Collard JG 1999 Rac downregulates Rho activity: reciprocal balance between both GTPases determines cellular morphology and migratory behavior. J Cell Biol 147:10091022

39. Yamaguchi Y, Katoh H, Yasui H, Mori K, Negishi M 2001 RhoA inhibits the nerve growth factor-induced Racl activation through Rho-associated kinase-dependent pathway. J Biol Chem 276:18977-18983 